\title{
Salazar-Knowles Equation Parameter B
}

National Cancer Institute

\section{Source}

National Cancer Institute. Salazar-Knowles Equation Parameter B. NCI Thesaurus. Code C139257.

The difference between the volume at total lung capacity and the hypothesized volume at a transpulmonary pressure of zero. 